\title{
BMJ Open Predictors of late presentation to renal dialysis: a cohort study of linked primary and secondary care records in East London
}

\author{
Ademola Olaitan, ${ }^{1}$ Neil Ashman, ${ }^{1}$ Kate Homer, ${ }^{2}$ Sally Hull ${ }^{\odot 2}$
}

To cite: Olaitan A, Ashman N, Homer $\mathrm{K}$, et al. Predictors of late presentation to renal dialysis: a cohort study of linked primary and secondary care records in East London. BMJ Open 2019;9:e028431. doi:10.1136/ bmjopen-2018-028431

- Pre-publication history and additional material is published online only. To view please visit the journal online (http://dx.doi. org/10.1136/bmjopen-2018028431).

Received 12 December 2018 Revised 26 March 2019 Accepted 21 May 2019

Check for updates

(c) Author(s) (or their employer(s)) 2019. Re-use permitted under CC BY-NC. No commercial re-use. See rights and permissions. Published by BMJ.

${ }^{1}$ Renal Medicine, Barts Health NHS Trust, London, UK

${ }^{2}$ Centre for Primary Care and Public Health, Queen Mary University of London, London, UK

Correspondence to

Sally Hull; s.a.hull@qmul.ac.uk

\section{ABSTRACT}

Objectives The outcomes and experience of care for patients who start renal replacement therapy (RRT) in an unplanned manner are worse than for those who have planned care. The objective of this study was to examine the primary care predictors of unplanned starts to RRT.

Design Retrospective cohort study with linked primary care and hospital data.

Setting 128 general practices in East London with a combined population of 1043346 people.

Participants 999 consecutive patients starting dialysis at Barts Health National Health Service Trust between September 2014 and August 2017.

Primary outcome measures Unplanned versus a planned start to dialysis among the cohort of 389 patients with a linked primary care record. An unplanned start to dialysis is defined as receiving nephrology care in the low clearance clinic (or equivalent) for less than 90 days. A planned start is defined as access to predialysis counselling and care for at least 90 days prior to commencing dialysis.

Results The adjusted logistic regression analysis showed that the most important modifiable risk factors for unplanned dialysis were the absence of a chronic kidney disease (CKD) code in the general practice (GP) record (OR $8.02,95 \% \mathrm{Cl} 3.65$ to 17.63) and the absence of prescribed lipid lowering medication (OR 2.37, $95 \% \mathrm{Cl} 1.05$ to 5.34 ). Other contributing factors included male gender and a greater number of long-term conditions.

Conclusions Improving CKD coding in primary care and the additional review and clinical scrutiny associated with this may contribute to a further reduction in unplanned RRT rates.

\section{INTRODUCTION}

A healthy or planned start to renal replacement therapy (RRT) requires patient education, peer support, shared decision making and where possible, preparation for pre-emptive transplantation. These elements of care are usually delivered in the setting of a dedicated multi-disciplinary clinic which comprises nephrologists, specialist nurses, dieticians and transplant and access surgeons. Patients who commence dialysis in an
Strengths and limitations of this study

- This study is the first to link UK primary care and hospital records to identify predictors of unplanned starts to dialysis.

- The study setting includes patients drawn from a multi-ethnic and socially deprived localities in London. Ethnicity recording in the linked data set was $99 \%$ complete.

- Follow-up for 1 year following dialysis ensured the capture of both early and medium term deaths.

- Linked data for analysis covered the entire population (900 000) of three London boroughs.

- We were unable to link primary care data from outlying London boroughs, reducing our population for analysis from 755 to 461

unplanned manner often lack these elements of care, and such starts have been associated with poor patient experience and outcomes.

Although there is no universally accepted definition of an unplanned dialysis start, Mendelssohn et al suggest common features include unscheduled hospitalisation at initiation of dialysis, lack of permanent vascular or peritoneal access for dialysis and an absence of patient choice. The lack of consensus on what constitutes an unplanned start most likely reflects different models of chronic kidney disease (CKD) care in different countries. In our population, we have defined unplanned dialysis as receiving less than 90 days follow-up by a nephrologist in a dedicated, multidisciplinary clinic for patients with an estimated glomerular filtration rate $($ eGFR $)<20 \mathrm{~mL} / \mathrm{min} / 1.73 \mathrm{~m}^{2} .{ }^{1}$

Unplanned dialysis is associated with up to fivefold more healthcare expenditure than a planned transition to dialysis. ${ }^{2}$ Patients starting in an unplanned manner also experience excess morbidity characterised by uraemic symptoms, fluid overload, increased blood transfusion requirements and increased 
frequency and duration of hospitalisation. ${ }^{23}$ Unplanned dialysis is also associated with a reduced opportunity to choose modality, and with reduced quality of life. ${ }^{45}$ Furthermore, multiple studies have demonstrated an association with increased short and medium term mortality in patients with unplanned dialysis starts. ${ }^{367}$

A significant proportion of patients commence dialysis following a late presentation although there has been a steady decline over the past ten years. The average rate of unplanned dialysis across the UK in 2016 was $15.6 \%$, with variation between centres ranging from $5 \%$ to $34 \% .{ }^{8}$

Over the study period 2014-2017 reviewing 1000 consecutive starts to dialysis, 39\% commenced in an unplanned manner, hence the urgent need to investigate and address this problem.

Despite the clinical and socioeconomic burden of unplanned dialysis, there has been little research into modifiable factors in primary care management in the period leading up to the start of dialysis. This study links the primary care record with hospital data to help identify the characteristics and primary care management of population groups who are at increased risk of an unplanned start to dialysis, and aims to identify opportunities to improve care.

\section{MATERIALS AND METHODS \\ Design and setting}

A retrospective cohort study to examine the primary care characteristics and antecedent care of patients who started dialysis at a specialist renal centre in East London using linked primary care and hospital data. The study was based in East London with the linked primary care data drawn from the three geographically contiguous East London borough-based Clinical Commissioning Groups (CCGs) in Newham, Tower Hamlets and City \& Hackney with a combined population of 1043346 people registered at 128 general practices. In the 2011 UK Census $48 \%$ of the population in these three CCGs was recorded to be of non-white ethnic origin, ${ }^{9}$ and the English indices of deprivation 2015 show that all three feature in the eight most socially deprived boroughs in England. ${ }^{10}$

The Department of Renal Medicine \& Transplantation at Barts Health National Health Service (NHS) Trust is the sole tertiary renal provider for North-East London, providing kidney care for over 1.84 million people across seven metropolitan boroughs with a high incident need for RRT. This ranges from 191 patients per million population (pmp) in the Borough of Newham to $91 \mathrm{pmp}$ in the Borough of Havering. ${ }^{8}$ This unit provides all in-patient and satellite dialysis facilities, home therapy, and a networked acute kidney injury (AKI) service across seven acute and specialist hospitals.

\section{Hospital audit data}

The audit data was collected retrospectively for 1000 consecutive cases (999 patients, with one case being a test record) starting dialysis between September 2014 and
August 2017, from the hospital dialysis records system (Renalware). Data included dialysis start date (index date), age at dialysis, gender, ethnicity, modality (haemodialysis or peritoneal dialysis), access type (temporary or tunnelled central venous catheter), arteriovenous fistula, arteriovenous graft and Moncrieff-Popovich or Tenckhoff peritoneal catheters), diagnosis at end stage renal failure, inpatient status at time of dialysis and eGFR (mL/ $\min / 1.73 \mathrm{~m}^{2}$ ) at dialysis start. Cases of AKI were identified by reviewing coded diagnoses and case records associated with each episode of care. Deceased data for each patient were collected from Renalware 1 year on from original extraction date to ensure adequate follow-up time.

\section{Linked primary care data}

The linked dataset (see figure 1) included all patients currently or previously registered at the 128 general practices on the extraction date in April 2018 who had NHS numbers matching those of the 999 patients in the audit sample. Primary care data were extracted for each patient prior to their first dialysis date (index date) from the North and East London Commissioning Support Unit which holds Egton Medical Information Systems (EMIS)-Web primary care data which can be linked to a patient audit sample using the encrypted NHS number. Data were extracted on secure N3 terminals using SQL Server Management Studio (2014). All data were anonymous and managed according to UK NHS information governance requirements.

\section{Sociodemographic}

Self-reported ethnicity was recorded at the practice during registration or routine consultation. Ethnic categories are based on the UK 2011 census and for this study were combined into three major categories: White (British, Irish, other white), black (black African, black Caribbean, black British, other black and mixed black), South Asian (Bangladeshi, Pakistani, Indian, Sri Lankan, British Asian, other South Asian or mixed Asian). We used the English indices of deprivation (IMD) 2015 score as a measure of social deprivation. This is the UK Government score of markers of socio-economic deprivation for small areas in England. We mapped the IMD score to each patient local super output area from 2011 and derived internal quintiles for our study population. ${ }^{10}$

\section{Clinical measures}

To assess multi-morbidity we extracted the presence of 17 quality and outcomes framework (QOF) long term conditions (LTCs), with an earliest recorded Read code prior to the index RRT date, using version 37 of the QOF business rule set. ${ }^{11}$ The conditions included were: asthma, atrial fibrillation, cancer, coronary heart disease, CKD, chronic obstructive pulmonary disease, dementia, depression, diabetes, epilepsy, heart failure, hypertension, learning disabilities, serious mental illness, osteoporosis, peripheral arterial disease and stroke and transient ischaemic attack. We used the total count of these QOF LTCs per 


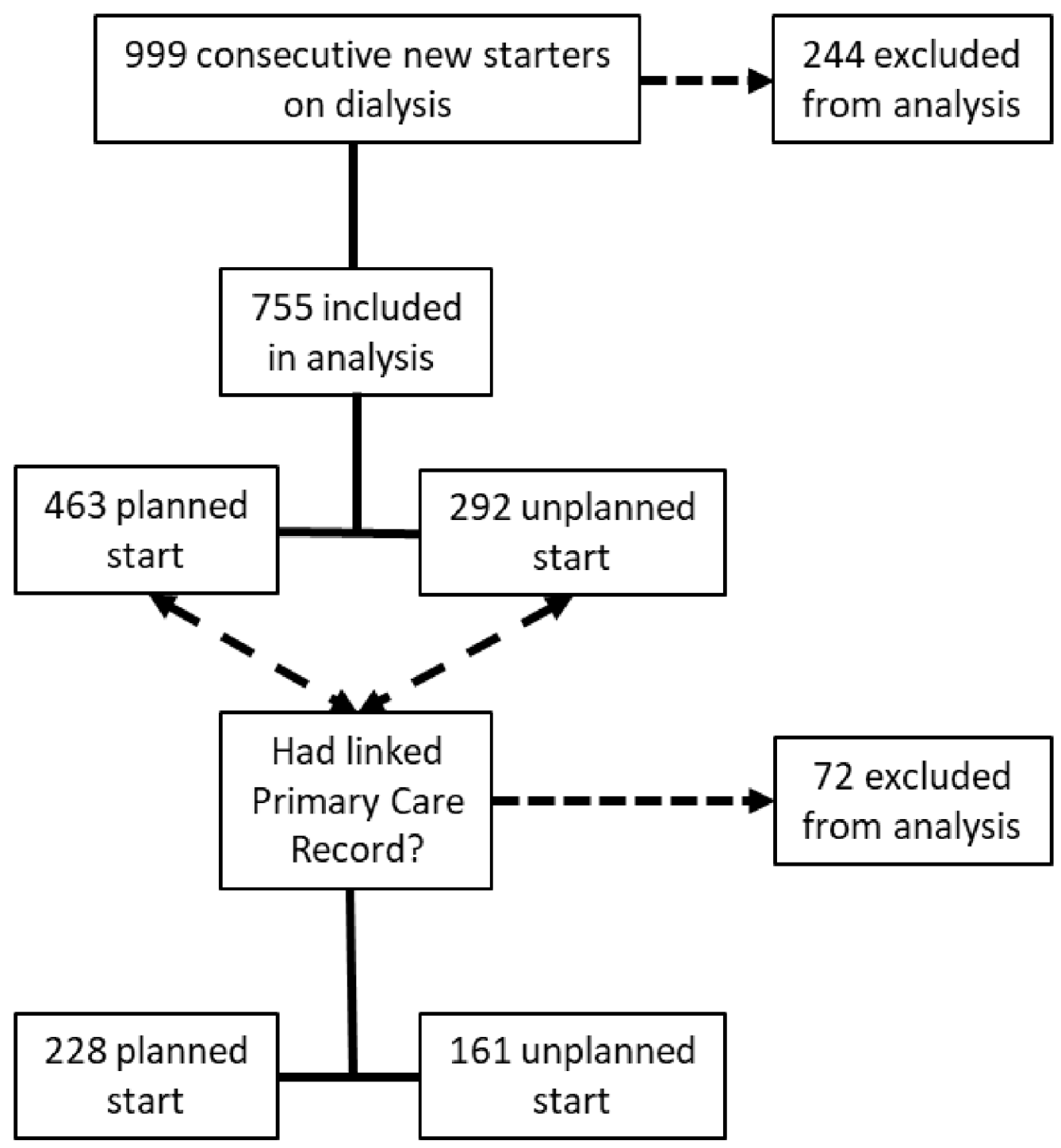

Figure 1 Primary care records linked to new starters on dialysis.

person, excluding CKD, as the principal measure of multimorbidity. ${ }^{12-14}$ We obtained data for presence of an influenza or pneumococcal vaccination in the year prior to index date and the presence of a hepatitis vaccination ever prior to the index date.

\section{Health service use}

We extracted a count of contacts with a general practitioner including surgery consultations, home visits and telephone contacts over a 2-year period prior to first dialysis date. This excluded contacts with nurses and other healthcare professionals as there is considerable variation in the assignment coding of non-medical consultations to different categories of user. ${ }^{15}$

\section{Prescribing}

We extracted prescriptions for ACE inhibitors or angiotensin II receptor blockers (ARBs), diuretics, other anti-hypertensives and statins in the 6 months prior to the index date. We also collected prescriptions for non-steroidal anti-inflammatory drug (NSAIDs) in the year prior to index date.

\section{Clinical tests}

We extracted a count of eGFR $\left(\mathrm{mL} / \mathrm{min} / 1.73 \mathrm{~m}^{2}\right)$ tests in the 5 years prior to index date, excluding tests with a value of $<5$. We extracted the latest ever values prior to index date for urine albumin creatinine ratio (uACR) $(\mathrm{mg} / \mathrm{mmol})$ and systolic blood pressure $(\mathrm{mm} \mathrm{Hg})$ and built a new variable 'target blood pressure achieved'. Target blood pressure was derived as systolic blood pressure $<130 \mathrm{~mm} \mathrm{Hg}$ for diabetics or non-diabetics with a uACR > 70; and as systolic blood pressure $<140 \mathrm{~mm} \mathrm{Hg}$ for non-diabetics with a uACR $\leq 70$ or uACR value is missing.

\section{STATISTICAL ANALYSIS}

\section{Outcomes}

Using a criteria classification system described in online supplementary appendix 1 all audit patients had been 
categorised into 'Planned', 'Unplanned' or 'Excluded'. Our primary dependent outcome was unplanned versus planned dialysis. We did not use the 'Excluded' patients in the model.

All statistical analysis was undertaken in Stata V.14 (StataCorp). We used univariate and multivariate logistic regression models adjusting for clusters in general practices.

Sensitivity analyses were undertaken to test the effect of:

1. Removing cases with AKI (recorded in the hospital record).

2. Removing cases with zero contact with a GP in the last 2 years.

3. Including diabetes, and hypertension without diabetes, as independent predictors.

\section{Patient and public involvement}

No patients or members of the public were involved in the design of this study.

\section{RESULTS}

\section{Hospital audit data}

The characteristics of the 999 consecutive hospital audit patients are shown in table 1 . The categorisation comprised planned starters $(\mathrm{n}=463)$, unplanned starters $(\mathrm{n}=292)$ and excluded cases $(\mathrm{n}=244)$ (see figure 1 and flow chart in online supplementary appendix 2). $85 \%$ of patients had recorded ethnicity, of whom $45 \%$ were black African/Caribbean or South Asian origin.

The patients in the planned group starting dialysis were older $(\mathrm{p}=0.014)$ and included slightly more females $(\mathrm{p}=0.045)$. The distribution of diabetes, hypertension and IgA nephropathy diagnoses were similar in both the planned and unplanned groups. Unplanned starters were more likely to be hospitalised at initiation of dialysis (OR 9.54, 95\% CI 6.78 to 13.41 ), and were significantly more likely to commence dialysis with temporary vascular access (OR 6.62, 95\% CI 4.48 to 9.80).

Over the complete follow-up time 217 (21.7\%) of the sample died. Of the $59(5.9 \%)$ who died within 90 days of starting RRT, 13 patients were in the unplanned group $(2.8 \%)$ and $12(4.1 \%)$ in the unplanned group. After adjusting for follow-up time after dialysis (at least 1 year), age at dialysis start and sex, patients in the unplanned group were twice as likely to die (OR 2.16,95\% CI 1.47 to 3.15) (data not shown).

\section{Linked primary care data}

We linked 461 (46\%) patients from the 999-strong hospital audit sample to patients registered in the East London CCGs of Newham 207 (44.9\%), Tower Hamlets $158(34.3 \%)$ and City \& Hackney $97(21.0 \%)$ (data not shown). Of the matched patients $72(15.6 \%)$ were excluded: 40 had AKI but became dialysis independent, 18 patients were out of the area, 10 had had previous transplants and four for other reasons (pregnancy, age under 18 , heart failure-see flow chart in online supplementary appendix 2). The patient characteristics of the linked dataset are shown in table 2.

\section{Sociodemographic}

The mean age of patients in the linked dataset was 57.9 $( \pm 15.2)$, and there were no statistically significant differences in age between the planned and unplanned groups. The proportion of females in the unplanned group was significantly less than the planned group $(p=0.008)$. Ethnicity recording in the linked primary care record was $99 \%$ complete, with $69 \%$ of those recorded of black African/Caribbean or South Asian origin.

\section{Clinical measures and tests}

There were similar proportions of patients with four or more LTCs in addition to CKD in both the planned and unplanned groups. The prevalence of diabetes was consistent across planned and unplanned groups but there were significantly more hypertensive patients in the planned group $(p=0.012)$. A blood pressure measurement in primary care was recorded for $96 \%$ of patients. Of those $38 \%$ had achieved target blood pressure, with similar proportions in both groups. There were significantly more patients with no eGFR in the last 5 years in the unplanned group, and conversely significantly more patients with six or more eGFR tests in the planned group.

\section{Health service use and prescribing}

The count of consultations with a GP in the 2 years prior to RRT was similar in both groups.

There were no differences in prescribing of ACE inhibitors or ARBs in the 6 months prior to RRT or NSAIDs in the year prior to RRT between planned and unplanned dialysis. In the planned group there were significantly more patients with statins, diuretics or other hypertensives.

\section{Multivariate analysis: primary care predictors of unplanned dialysis}

The multivariate model presented in table 3 is the primary regression model, with the sensitivity analyses on it. This model shows that the most important independent modifiable factors, adjusted for all others in the table, that predict unplanned dialysis are the lack of a CKD Read code (OR 8.13, 95\% CI 3.74 to 17.67), and the absence of statin prescribing (OR 2.37, 95\% CI 1.05 to 5.34). Other factors that predict unplanned dialysis include male sex, the absolute count of a patient's LTCs and an absence of hepatitis $\mathrm{B}$ vaccination.

\section{Sensitivity analyses}

1. Exploring the effect of excluding those with AKI. These events may occur in the context of a severe intercurrent illness, and may be unpredictable and hence not amenable to prevention. When these cases were excluded $(n=42)$ the model did not change except that absence of statins was no longer a significant predictor (online supplementary appendix 3 ). 
Table 1 Characteristics of hospital audit population

\begin{tabular}{|c|c|c|c|c|}
\hline & All & Planned & Unplanned & Exclusions \\
\hline N (\%) & $999(100)$ & $463(46.4)$ & 292 (29.2) & $244(24.4)$ \\
\hline \multicolumn{5}{|l|}{ Gender } \\
\hline Male & $626(62.7)$ & $272(58.7)$ & $193(66.1)$ & $161(66.0)$ \\
\hline Female & 373 (37.3) & 191 (41.3) & 99 (33.9) & $83(34.0)$ \\
\hline Mean (SD) age, years & $57.3(15.4)$ & $57.9(14.9)$ & $55.1(15.6)$ & $58.9(16.0)$ \\
\hline \multicolumn{5}{|l|}{ Ethnicity } \\
\hline White & $268(26.8)$ & $125(27.0)$ & $78(26.7)$ & $65(26.6)$ \\
\hline South Asian & 233 (23.3) & $121(26.1)$ & $66(22.6)$ & $46(18.9)$ \\
\hline Black & $154(15.4)$ & 75 (16.2) & $39(13.4)$ & $40(16.4)$ \\
\hline Other & $197(19.7)$ & $88(19.0)$ & $67(22.9)$ & $42(17.2)$ \\
\hline Unknown * & $147(14.7)$ & $54(11.7)$ & $42(14.4)$ & $51(20.9)$ \\
\hline \multicolumn{5}{|l|}{ Deceased } \\
\hline Over all follow-up & $217(21.7)$ & $69(14.9)$ & $75(25.7)$ & 73 (29.9) \\
\hline Within 90 days RRT & $59(5.9)$ & $13(2.8)$ & $12(4.1)$ & 34 (13.9) \\
\hline \multicolumn{5}{|l|}{ Inpatient at RRT start } \\
\hline No & $440(44.0)$ & 356 (76.9) & $75(25.7)$ & $9(3.7)$ \\
\hline Yes & $433(43.3)$ & $106(22.9)$ & $213(72.9)$ & $114(46.7)$ \\
\hline Unknown & $126(12.6)$ & $1(0.2)$ & $4(1.4)$ & $121(49.6)$ \\
\hline \multicolumn{5}{|l|}{ Modality } \\
\hline HD & 728 (72.9) & $279(60.3)$ & 231 (79.1) & 219 (89.8) \\
\hline PD & $270(27.0)$ & $184(39.7)$ & $61(20.9)$ & $25(10.2)$ \\
\hline \multicolumn{5}{|l|}{ Access } \\
\hline CVC & 277 (27.7) & $43(9.3)$ & $118(40.4)$ & $116(47.5)$ \\
\hline Tunnelled line & $249(24.9)$ & $103(22.2)$ & $117(40.1)$ & $29(11.9)$ \\
\hline AVF/AVG & $150(15.0)$ & $132(28.5)$ & $3(1.0)$ & $15(6.1)$ \\
\hline Tenckhoff & $210(21.0)$ & $152(32.8)$ & $53(18.2)$ & $5(2.0)$ \\
\hline Moncrief & $35(3.5)$ & $33(7.1)$ & $1(0.3)$ & $1(0.4)$ \\
\hline Missing data & $78(7.8)$ & 0 & 0 & $78(32.0)$ \\
\hline \multicolumn{5}{|c|}{ Diagnosis from hospital record at RRT } \\
\hline Diabetes & $287(28.7)$ & $134(28.9)$ & $90(30.8)$ & $63(25.8)$ \\
\hline IgA nephropathy & $47(4.7)$ & $24(5.2)$ & $16(5.5)$ & $7(2.9)$ \\
\hline Hypertension & $46(4.6)$ & $23(5.0)$ & $15(5.1)$ & $8(3.3)$ \\
\hline Polycystic kidney & $34(3.4)$ & $15(3.2)$ & $6(2.1)$ & $13(5.3)$ \\
\hline Glomerulonephritis & $26(2.6)$ & $14(3.0)$ & $5(1.7)$ & $7(2.9)$ \\
\hline Missing data & $297(29.7)$ & $117(25.3)$ & $79(27.1)$ & $101(41.4)$ \\
\hline $\begin{array}{l}\text { Acute kidney injury } \\
\text { identified from hospital } \\
\text { record }\end{array}$ & 196 & 1 & 72 & 123 \\
\hline \multicolumn{5}{|l|}{ eGFR at RRT start } \\
\hline Median (IQR) & $8(4)$ & $8(4)$ & $8(4)$ & $8(5)$ \\
\hline Missing data & $20(2.0)$ & $5(1.1)$ & $7(2.4)$ & $8(3.3)$ \\
\hline
\end{tabular}

*Unknown ethnic group=not stated or missing.

AVF, arteriovenous fistula; AVG, arteriovenous graft; CVC, central venous catheter; eGFR, estimated glomerular filtration rate; HD, haemodialysis; PD, peritoneal dialysis; RRT, renal replacement therapy. 
Open access

Table 2 Characteristics of linked dataset from primary care records

\begin{tabular}{|c|c|c|c|c|}
\hline & All & Planned & Unplanned & Exclusions \\
\hline $\mathrm{N}(\%)$ & $461(100)$ & $228(49.5)$ & $161(34.9)$ & $72(15.6)$ \\
\hline \multicolumn{5}{|l|}{ Gender } \\
\hline Male & 292 (63.3) & $136(59.6)$ & $120(72.7)$ & $39(54.2)$ \\
\hline Female & $169(36.7)$ & $92(40.4)$ & $44(27.3)$ & $33(45.8)$ \\
\hline \multicolumn{5}{|l|}{ Age band } \\
\hline$<18$ & $1(0.2)$ & 0 & 0 & $1(1.4)$ \\
\hline $18-54$ & $172(37.3)$ & 75 (32.9) & $71(44.1)$ & $26(36.1)$ \\
\hline $55-74$ & $220(47.7)$ & $123(53.9)$ & $67(41.6)$ & $30(41.7)$ \\
\hline $75+$ & $68(14.8)$ & $30(13.2)$ & $23(14.3)$ & $15(20.8)$ \\
\hline \multicolumn{5}{|l|}{ Ethnicity } \\
\hline White & $125(27.1)$ & $42(18.4)$ & 52 (32.3) & $31(43.1)$ \\
\hline South Asian & $182(39.5)$ & $104(45.6)$ & $58(36.0)$ & $20(27.8)$ \\
\hline Black & $134(29.1)$ & $73(32.0)$ & $45(28.0)$ & $16(22.2)$ \\
\hline Other & $15(3.3)$ & $6(2.6)$ & $5(3.1)$ & $4(5.6)$ \\
\hline Unknown* & $5(1.1)$ & $3(1.3)$ & $1(0.6)$ & $1(1.4)$ \\
\hline \multicolumn{5}{|c|}{ Study population IMD 2015 quintiles } \\
\hline 1 (least deprived) & $95(20.6)$ & $35(15.4)$ & $44(27.3)$ & $16(22.2)$ \\
\hline 2 & $90(19.5)$ & $51(22.4)$ & $26(16.1)$ & $13(18.1)$ \\
\hline 3 & $92(20.0)$ & $43(18.9)$ & $33(20.5)$ & $16(22.2)$ \\
\hline 4 & $93(20.2)$ & $39(17.1)$ & $39(24.2)$ & $15(20.8)$ \\
\hline 5 (most deprived) & $91(19.7)$ & $60(26.3)$ & $19(11.8)$ & $12(16.7)$ \\
\hline \multicolumn{5}{|l|}{ Deceased } \\
\hline Over all follow-up & $106(23.0)$ & $34(14.9)$ & $50(31.1)$ & $22(30.6)$ \\
\hline Within 90 days RRT & $30(6.5)$ & $8(3.5)$ & $18(11.2)$ & $4(5.6)$ \\
\hline \multicolumn{5}{|c|}{ Chronic kidney disease coded prior to RRT } \\
\hline Yes & $339(73.5)$ & $212(93.0)$ & $96(59.6)$ & $31(43.1)$ \\
\hline \multicolumn{5}{|c|}{ AKI (from the hospital record) } \\
\hline Yes & 87 & 1 & 42 & 44 \\
\hline \multicolumn{5}{|c|}{ Hypertension with no diabetes coded prior to RRT } \\
\hline Yes & $125(27.1)$ & $66(28.9)$ & $42(26.1)$ & $17(23.6)$ \\
\hline \multicolumn{5}{|c|}{ Diabetes coded prior to RRT } \\
\hline Yes & $255(55.3)$ & $136(59.6)$ & $85(52.8)$ & $34(47.2)$ \\
\hline \multicolumn{5}{|c|}{ Count of long-term conditions excluding CKD } \\
\hline 0 & $46(10.0)$ & $12(5.3)$ & $18(11.2)$ & $16(22.2)$ \\
\hline 1 & $112(24.3)$ & $61(26.8)$ & $35(21.7)$ & $16(22.2)$ \\
\hline 2 & $108(23.4)$ & $59(25.9)$ & $38(23.6)$ & $11(15.3)$ \\
\hline 3 & $93(20.2)$ & $51(22.4)$ & $34(21.1)$ & $8(11.1)$ \\
\hline $4+$ & $102(22.1)$ & $45(19.7)$ & $36(22.4)$ & $21(29.2)$ \\
\hline \multicolumn{5}{|c|}{ eGFR tests in the 5 years prior to RRT } \\
\hline Median (IQR) & $6(8)$ & $7(9)$ & $5(8)$ & $4(6)$ \\
\hline $1-5$ & $151(32.8)$ & $67(29.4)$ & $54(33.5)$ & $30(41.7)$ \\
\hline 0 & $57(12.4)$ & $17(7.5)$ & 27 (16.8) & $13(18.1)$ \\
\hline$\geq 6$ & $253(54.9)$ & $144(63.2)$ & $80(49.7)$ & $29(40.3)$ \\
\hline \multicolumn{5}{|c|}{ Reaching target BP ever prior to RRT } \\
\hline Yes & $173(37.5)$ & $78(34.2)$ & $58(36.0)$ & $37(51.4)$ \\
\hline
\end{tabular}

Continued 
Table 2 Continued

\begin{tabular}{|c|c|c|c|c|}
\hline & All & Planned & Unplanned & Exclusions \\
\hline Missing data & $17(3.7)$ & $5(2.2)$ & $9(5.6)$ & $3(4.2)$ \\
\hline \multicolumn{5}{|c|}{ GP consultations prior to RRT $\dagger$} \\
\hline In the previous year & $394(85.5)$ & $202(88.6)$ & $131(81.4)$ & $61(84.7)$ \\
\hline $\begin{array}{l}\text { Median (IQR) count in } \\
\text { previous } 2 \text { years }\end{array}$ & $12(14)$ & $13(14)$ & $11(17)$ & $11(15)$ \\
\hline \multicolumn{5}{|c|}{ Influenza or pneumococcal vaccination in the year prior to RRT } \\
\hline Yes & $279(60.5)$ & $162(71.1)$ & $80(49.7)$ & $37(51.4)$ \\
\hline \multicolumn{5}{|c|}{ Hepatitis B vaccination ever prior to RRT } \\
\hline Yes & $119(25.8)$ & $105(46.1)$ & $10(6.2)$ & $4(5.6)$ \\
\hline \multicolumn{5}{|c|}{ Prescribing in the 6 months prior to RRT } \\
\hline ACEI or ARB & $199(43.2)$ & $87(38.2)$ & $73(45.3)$ & $39(54.2)$ \\
\hline Diuretics & $269(58.4)$ & $161(70.6)$ & $77(47.8)$ & $31(43.1)$ \\
\hline Other hypertensives $\ddagger$ & $346(75.1)$ & $196(86.0)$ & $105(65.2)$ & $45(62.5)$ \\
\hline Statins & $281(61.0)$ & $161(70.6)$ & $78(48.4)$ & $42(58.3)$ \\
\hline \multicolumn{5}{|c|}{ NSAIDs in the year prior to RRT } \\
\hline Yes & $28(6.1)$ & $10(4.4)$ & $11(6.8)$ & $7(9.7)$ \\
\hline
\end{tabular}

*Unknown ethnic group=not stated or missing.

†Face to face in surgery, telephone consultation or home visit (home or care home).

$\ddagger$ Vasodilators, Centrally acting anti-hypertensives, Alpha adrenoceptor blockers, Calcium channel blockers, Beta adrenoceptor blockers. ARB, angiotensin II receptor blockers; BP, blood pressure; CKD, chronic kidney disease; eGFR, estimated glomerular filtration rate; IMD, Index of Multiple Deprivation; NSAID, non-steroidal anti inflammatory drug; RRT, renal replacement therapy (dialysis).

2. When patients with zero contact with their GP in the 2 years prior to dialysis are excluded $(n=20)$ the count of LTCs and absence of statins are no longer significant predictors (online supplementary appendix 3).

3. When diabetes and hypertension with no diabetes are included as independent predictors the count of LTCs is no longer significant (online supplementary appen$\operatorname{dix} 3)$.

\section{DISCUSSION}

\section{Main findings}

This is one of the largest retrospective studies to compare the outcomes of planned and unplanned starts to dialysis, and to use linked primary care data to seek modifiable predictors of late presenters to dialysis. From the hospital audit data, we find that unplanned dialysis starts are associated with twice the mortality rate of planned starts over the 1-year follow-up period. The cohort is younger (mean age 57.3) when compared with national data from the UK Renal Registry for 2016, but has a similar prevalence of diabetes to the national cohort. ${ }^{8}$

The linked primary care data for 461 cases demonstrates that the most important modifiable factor predictive of unplanned dialysis is the absence of a diagnostic Read code for CKD. This finding appears to be robust, the sensitivity analyses which removed cases admitted with AKI and cases with no GP consultations in the previous 2 years did not alter the strength of this association (see online supplementary appendix 3 ).
National Institute for Health and Care Excellence guidance $^{16}$ recommends regular testing for CKD in primary care for those at high risk of CKD such as patients with hypertension, CVD and diabetes. The addition of a diagnostic Read code to the patient record enables regular recall and safer prescribing decisions. The national CKD audit in primary care found that less than $70 \%$ of CKD, identifiable from eGFR values, had an associated CKD Read code in the electronic health record. ${ }^{17}$

We found no difference by ethnicity in the adjusted analysis, but as expected the risk of unplanned dialysis rises with a greater number of patient comorbidities. The finding that statin prescribing is associated with planned dialysis can be linked to the findings on diagnostic coding. Previous work has demonstrated that the presence of a diagnostic CKD code in primary care is associated with better clinical management, including a greater likelihood of BP managed to target, statin prescription and regular preventive immunisation. ${ }^{18} 19$

These findings suggest that the additional scrutiny which is triggered by diagnostic coding may provide patients with a better chance of avoiding a late referral to low clearance clinics.

\section{Implications for clinical practice}

Although the UK Renal Registry reports a single category for unplanned starts to dialysis, it is well recognised that the causes of late presentation are complex. ${ }^{8}$ 
Table 3 Model for predictors of unplanned dialysis over planned dialysis, $n=375$ cases contributing to model

$\begin{array}{llll}\text { Univariate OR } & \mathbf{P} & 95 \% \mathrm{Cl} & \text { Multivariate OR* }\end{array}$

$95 \% \mathrm{CI}$

\begin{tabular}{|c|c|c|c|c|c|c|}
\hline \multicolumn{7}{|l|}{ Gender } \\
\hline Male (ref) & 1 & & & 1 & & \\
\hline Female & 0.56 & 0.00 & (0.38 to 0.82$)$ & 0.49 & 0.01 & (0.29 to 0.84$)$ \\
\hline $18-54$ (ref) & 1 & & & 1 & & \\
\hline $55-74$ & 0.58 & 0.03 & (0.35 to 0.93$)$ & 0.94 & 0.84 & $(0.49$ to 1.80$)$ \\
\hline \multicolumn{7}{|l|}{ Ethnicity† } \\
\hline White (ref) & 1 & & & 1 & & \\
\hline South Asian & 0.45 & 0.00 & $(0.27$ to 0.76$)$ & 0.58 & 0.11 & (0.30 to 1.12$)$ \\
\hline Black & 0.50 & 0.01 & (0.29 to 0.85$)$ & 0.58 & 0.17 & $(0.27$ to 1.25$)$ \\
\hline \multicolumn{7}{|c|}{ Count of long term conditions excluding CKD } \\
\hline 2 & 0.43 & 0.06 & (0.18 to 1.03 ) & 3.52 & 0.06 & (0.93 to 13.32$)$ \\
\hline 3 & 0.44 & 0.05 & (0.20 to 0.99 ) & 3.71 & 0.04 & (1.05 to 13.14$)$ \\
\hline $4+$ & 0.53 & 0.16 & (0.22 to 1.28$)$ & 4.34 & 0.04 & (1.05 to 18.02$)$ \\
\hline
\end{tabular}

Chronic kidney disease coded prior to RRT

\begin{tabular}{|c|c|c|c|c|c|c|}
\hline Yes (ref) & 1 & & & 1 & & \\
\hline No & 8.97 & 0.00 & (5.41 to 14.87$)$ & 8.13 & 0.00 & (3.74 to 17.67$)$ \\
\hline \multicolumn{7}{|c|}{ Prescribed statins in the 6 months prior to RRT } \\
\hline Yes (ref) & 1 & & & 1 & & \\
\hline No & 2.56 & 0.00 & (1.71 to 3.83$)$ & 2.37 & 0.04 & (1.05 to 5.34$)$ \\
\hline
\end{tabular}

eGFR tests in the last 5 years prior to RRT

$\begin{array}{lllllll}1-5 \text { (ref) } & 1 & & 1 & 0.76 & (0.26 \text { to } 2.72) \\ 0 & 1.97 & 0.04 & (1.03 \text { to } 3.78) & 0.83 & 0.89 & (0.50 \text { to } 1.83)\end{array}$

Reaching target BP ever prior to RRT

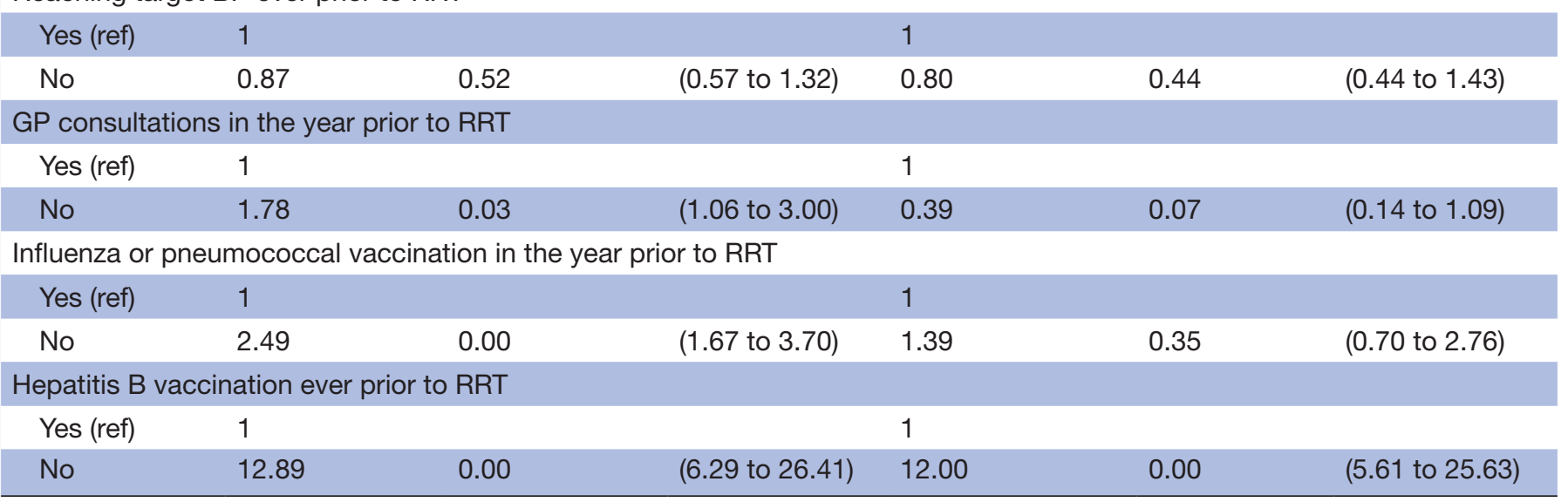

*Adjusted for other variables in the table and Index of Multiple Deprivation 2015.

†Other' and 'Unknown' ethnic group categories not shown.

CKD, chronic kidney disease; eGFR, estimated glomerular filtration rate; RRT, renal replacement therapy.

Udayaraj et $a l^{20}$ identify that this single definition encompasses cases with irreversible AKI which are rarely possible to anticipate either in primary care or in secondary care chronic disease clinics. By restricting the term late referral (among late presenters) to those without AKI, the numbers of patients with missed 
opportunities for earlier intervention are much reduced.

Efforts to reduced unplanned dialysis start should be directed at this cohort. Patients may fall into this group for multiple reasons including sudden rapid decline, patient-related factors such as non-engagement, migration or language barriers. Service related factors such as delayed time from referral to consultation will also play a part.

Service interventions to reduce late referrals to dialysis need to start in primary care settings. Our data suggest a key area for intervention is to ensure that once CKD is detected it should also be coded. Further interventions should encourage the systematic review of all patients with CKD promoted by national guidelines-with particular attention to blood pressure treatment, cardiovascular risk management and safer prescribing, ${ }^{16}$ and a greater awareness of population groups at risk of rapid progression. ${ }^{21}$ Such initiatives involve both practice-facing quality improvement programmes and laboratory based schemes to identify those at risk of progression based on tracking changes in eGFR values. $^{22-24}$

\section{Strengths and limitations}

The strengths of this study include the size of the dialysis cohort and the value gained by linking the hospital and primary care records. As the study was set in a multiethnic area we could also examine the effect of ethnicity on rates of unplanned dialysis.

Limitations of the study include the lack of primary care data from some of the boroughs contributing to the hospital dialysis cohort. These areas have a similar demography to the inner East London boroughs, but the possibility of unexplained bias within the data remains.

There are well known limitations to the use of routinely collected clinical data. There will inevitably be inaccuracies and omissions in the clinical data set. However, recording of diagnosis and the clinical process of care in the Quality and Outcomes domains are likely to be accurate.

A further limitation is that the data is drawn from one area of London, and from the records of one nephrology provider unit. Although the study population is not representative of the UK as a whole, the findings are generalisable to many urban areas throughout the UK which have a similar population profile.

\section{CONCLUSIONS}

Unplanned initiation of dialysis remains an important clinical challenge which is associated with excess mortality and morbidity, and is resource intensive. This study confirms the higher risk of death for this group. Findings in the linked primary care data suggest that interventions to improve rates of diagnostic CKD coding, and the regular surveillance associated with this, may contribute to reducing late referrals.
Acknowledgements The authors are grateful to the participating GPs for their cooperation, without which such studies would be impossible.

Contributors The study was devised by SH and NA, AO extracted the hospital data, $\mathrm{KH}$ undertook all data analysis. All authors contributed to writing and review of the report.

Funding This study was supported by an Innovating for Improvement grant from the Health Foundation.

Competing interests None declared.

Patient consent for publication Not required.

Provenance and peer review Not commissioned; externally peer reviewed. Data sharing statement № additional data are available.

Open access This is an open access article distributed in accordance with the Creative Commons Attribution Non Commercial (CC BY-NC 4.0) license, which permits others to distribute, remix, adapt, build upon this work non-commercially, and license their derivative works on different terms, provided the original work is properly cited, appropriate credit is given, any changes made indicated, and the use is non-commercial. See: http://creativecommons.org/licenses/by-nc/4.0/.

\section{REFERENCES}

1. Mendelssohn DC, Malmberg C, Hamandi B. An integrated review of "unplanned" dialysis initiation: reframing the terminology to "suboptimal" initiation. BMC Nephrol 2009;10:22.

2. Górriz JL, Sancho A, Pallardó LM, et al. [Prognostic significance of programmed dialysis in patients who initiate renal substitutive treatment. Multicenter study in Spain]. Nefrologia 2002;22:49-59.

3. Castellano I, Gallego S, Labrador PJ, et al. [The start of dialysis treatment in a Spanish department]. Nefrologia 2006;26:379-81.

4. Marrón B, Ortiz A, de Sequera P, et al. Impact of end-stage renal disease care in planned dialysis start and type of renal replacement therapy - a Spanish multicentre experience. Nephrology Dialysis Transplantation 2006;21(suppl 2):ii51-ii55.

5. Caskey FJ, Wordsworth S, Ben T, et al. Early referral and planned initiation of dialysis: what impact on quality of life? Nephrol Dial Transplant 2003;18:1330-8.

6. Roy D, Chowdhury AR, Pande S, et al. Evaluation of unplanned dialysis as a predictor of mortality in elderly dialysis patients: a retrospective data analysis. BMC Nephrol 2017;18:364.

7. Bradbury BD, Fissell RB, Albert JM, et al. Predictors of early mortality among incident US hemodialysis patients in the Dialysis Outcomes and Practice Patterns Study (DOPPS). Clin J Am Soc Nephrol 2007;2:89-99.

8. Hole B, Gilg J, Casula A, et al. Chapter 1 UK Renal Replacement Therapy Adult Incidence in 2016: National and Centre-specific Analyses. Nephron 2018;139(Suppl 1):13-46.

9. Office for National Statistics. Census 2011. Ethnic Group, Usual Resident. KS201EW 2011 http://www.nomisweb.co.uk/census/2011/ ks201ew.

10. Department for Communities and Local Government. English indices of deprivation. 2015;2015 https://www.gov.uk/government/statistics/ english-indices-of-deprivation-2015.

11. Digital NHS. Business Rules for Quality and Outcomes Framework (QOF): NHS Digital. 2015 http://content.digital.nhs.uk/qofbrv33.

12. Brilleman SL, Gravelle H, Hollinghurst S, et al. Keep it simple? Predicting primary health care costs with clinical morbidity measures. $J$ Health Econ 2014;35:109-22.

13. Barnett K, Mercer SW, Norbury M, et al. Epidemiology of multimorbidity and implications for health care, research, and medical education: a cross-sectional study. Lancet 2012;380:37-43.

14. Hull SA, Homer K, Boomla K, et al. Population and patient factors affecting emergency department attendance in London: retrospective cohort analysis of linked primary and secondary care records. $\mathrm{Br} \mathrm{J}$ Gen Pract 2018;68:e157-e167.

15. Hull SA, McKibben S, Homer K, et al. Asthma prescribing, ethnicity and risk of hospital admission: an analysis of 35,864 linked primary and secondary care records in East London. NPJ Prim Care Respir Med 2016;26:16049.

16. National Institute for Health and Care Excellence. Chronic kidney disease in adults: assessment and management. nice.org.uk/ guidance/cg182.2014.

17. Nitsch D, Caplin B, Hull SA, et al. National Chronic Kidney Disease Audit: National Report (Part 1). 2017 http://www.ckdaudit.org.uk/ files/4614/8429/6654/08532_CKD_Audit_Report_Jan_17_FINAL.pdf;. 
18. Kim LG, Cleary F, Wheeler DC, et al. How do primary care doctors in England and Wales code and manage people with chronic kidney disease? Results from the National Chronic Kidney Disease Audit. Nephrol Dial Transplant 2018;33:1373-1379.

19. Jain P, Calvert M, Cockwell P, et al. The need for improved identification and accurate classification of stages 3-5 Chronic Kidney Disease in primary care: retrospective cohort study. PLoS One 2014;9:e100831.

20. Udayaraj UP, Haynes R, Winearls CG. Late presentation of patients with end-stage renal disease for renal replacement therapy--is it always avoidable? Nephrol Dial Transplant $2011 ; 26: 3646-51$

21. Mathur R, Dreyer G, Yaqoob MM, et al. Ethnic differences in the progression of chronic kidney disease and risk of death in a UK diabetic population: an observational cohort study. BMJ Open 2018;8:e020145.

22. Rayner HC, Baharani J, Dasgupta I, et al. Does community-wide chronic kidney disease management improve patient outcomes? Nephrol Dial Transplant 2014;29:644-9.

23. Stevens PE, O'Donoghue DJ, de Lusignan S, et al. Chronic kidney disease management in the United Kingdom: NEOERICA project results. Kidney Int 2007;72:92-9.

24. Gallagher H, Methven S, Casula A, et al. A programme to spread eGFR graph surveillance for the early identification, support and treatment of people with progressive chronic kidney disease (ASSIST-CKD): protocol for the stepped wedge implementation and evaluation of an intervention to reduce late presentation for renal replacement therapy. BMC Nephrol 2017;18:131. 